\title{
A New High-Precision SCARA Design and Experiment
}

\author{
Xiaohui Xie ${ }^{1, a, *}, \mathrm{Ge} \mathrm{Li}^{2, \mathrm{~b}}$, lining Sun ${ }^{1, \mathrm{c}}$ \\ ${ }^{1}$ Robotics and Microsystems center, Soochow University, Soochow, P. R. China \\ ${ }^{2}$ School of Continuing Education, Shenzhen Polytechnic, Shenzhen, P. R. China \\ axhxie@suda.edu.cn, blige@szpt.edu.cn, 'Insun@suda.edu.cn \\ *Corresponding Author
}

Keywords: SCARA, speed, precision, assemblage

\begin{abstract}
This paper first described the composition of the SCARA robot, which contains the mechanical structures, drive systems and control systems. According to its mechanical structure, the mathematical model for SCARA is established by the D-H method, and then forward kinematics and inverse kinematics is deduced. Secondly, linear and circular interpolation method is researched in the Cartesian space, cubic polynomial interpolation and cubic spline interpolation method is researched in the joint space. Work in image-grasping, robot feeding and unloading is made by using the designed SCARA in electronic factory. Then the good results are got in such work.
\end{abstract}

\section{Introduction}

Now, many kinds of robot have been used in the industry production over the world, including robot in rectangular coordinates, robot in cylindrical coordinates, and robot in joint coordinates. There are vertical joint robot and horizontal joint robot which are included in the robot of joint coordinates, that the horizontal joint robot is most used in the industry production.

SCARA (Selective Compliance Assembly Robot Arm) is a kind of horizontal joint robot. Because simple mechanical design, high speed, high precision and good flexibility, SCARA have been widely used in industry production, mostly be used in assembling manufacturing. Some company have produced SCARA like YK-X version SCARA made by Yamaha company and EPSON-G made by Epson company in Japan. YK-400XG is a specific type robot with $\pm 0.01 \mathrm{~mm}$ repeat position accuracy in $\mathrm{X} / \mathrm{Y} / \mathrm{Z}$ axis and $\pm 0.05^{\circ}$ in $\mathrm{R}$ axis. The reciprocating cycle time of this robot is 0.48 second. The high-speed and high-precision SCARA (Model number: HK400) is designed that its reciprocating cycle time is 0.45 second.

SCARA can be used in manufacturing, welding, sealing, handling and pick-and-place with highefficiency need. SCARA spends shorter time than multi-axis positioning platform in rectangular coordinates if doing the same job.

A lot of researchers concentrate on the kinematics theory of SCARA, with such results are enough for position control like handling, feeding and unloading, dispensing work [1-3]. There are a lot of application which need force control like grinding, polishing and screw twisting. In such cases, the research on dynamics is needed. This paper will introduce a new designed SCARA and concentrate on new viewpoint of kinematics research.

\section{Robot Design}

\subsection{System design}

The mechanical structure of SCARA is composed of robot base, big arm, small arm, lifter and end effector. Robot base is used to support big arm and bear drive units. Big arm and small arm are two axes which can realize the designed range of movement. Lifter bears the end effector and it can realize up-down movement and rotating movement by using a spline rod. SCARA has three rotating axes and one straight axis. The axle wire of three axes are parallel and it can easy to reach one point 
in a plane. The straight axis can realize the up-down movement in $\mathrm{Z}$ axis.
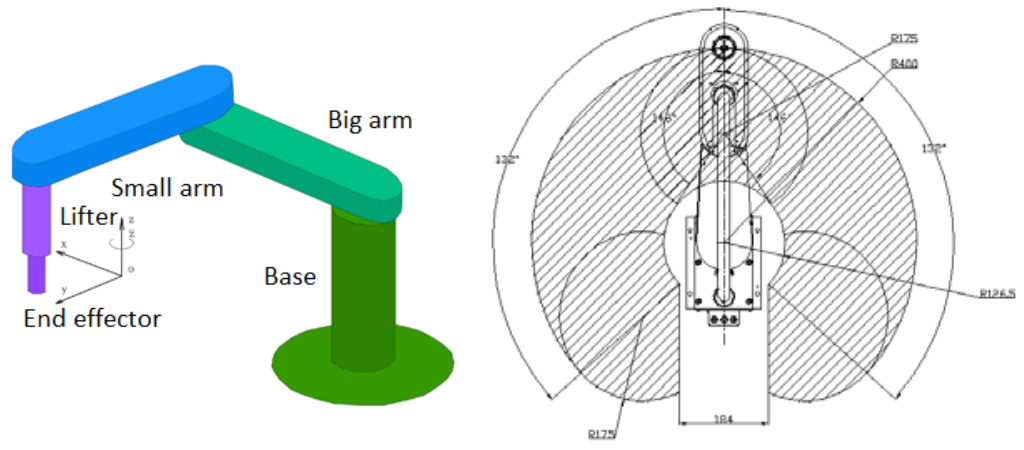

Figure 1 The robot structure of the designed SCARA

The working range of the designed SCARA is shown as Figure 1. The SCARA can work in a radius of about 400 millimeter over an angle of about 264 degree in working plane.

The traditional robot control system usually use independent control module and drive module. There are a lot of electrical lines that need to connect the control module and drive module. The separation between control and driving part leads to high cost and hard to maintenance. The designed SCARA adopts drive-control integration system, which has merits like easy connection and convenient maintenance.

\subsection{Robot kinematics analysis}

According to the knowledge of robot kinematics, the D-H (Denavit-Hartenberg) law is adopted to analyze the designed SCARA [4,5]. The Connecting rod coordinate system for SCARA like Fig. 1 is set up according to the steps as follows.

(1) Zero point $O_{n}$ is that the intersection point defines common perpendicular of joint axis between $J_{n}$ and $J_{n+1}$ on axis of $J_{n}$.

(2) Axis $Z_{n}$ is the same direction as the axis of joint $J_{n}$.

(3) Axis $X_{n}$ is the same direction as the common perpendicular of $J_{n}$ and $J_{n+1}$ from the point $O_{n}$ to the joint $J_{n+1}$.

(4) Axis $Y_{n}$ is the direction according to Axis $Z_{n}$ and Axis $X_{n}$ by the right-hand rule.

(5) Joint angle $\theta_{n}$ is the angle moving around Axis $Z_{n}$ from $X_{n-1}$ to $X_{n}$.

(6) Rod offset $d_{n}$ is the offset moving along Axis $Z_{n}$ from $X_{n-1}$ to $X_{n}$.

(7) Rod length $a_{n-1}$ is the length moving along Axis $X_{n-1}$ from $Z_{n-1}$ to $Z_{n}$.

(8) Rod twist angle $\alpha_{n-1}$ is the angle moving around Axis $X_{n-1}$ from $Z_{n-1}$ to $Z_{n}$.

Table. 1 D-H value of the designed SCARA

\begin{tabular}{|c|c|c|c|c|}
\hline Connecting rod & $\boldsymbol{\alpha}_{\mathrm{n}-1}$ & $a_{n-1}$ & $\Theta_{n}$ & $d_{n}$ \\
\hline 1 & 0 & 0 & $\Theta_{1}$ & $d_{1}$ \\
\hline 2 & 0 & 200 & $\Theta_{2}$ & 0 \\
\hline 3 & 180 & 200 & 0 & $d_{3}$ \\
\hline 4 & 0 & 0 & $\Theta_{4}$ & 0 \\
\hline
\end{tabular}

By such definition, the corresponding initial value and parameter of the designed SCARA is shown in Table.1. The general formula of homogeneous transformation is described as equation (1) that in this equation $c=\cos$ and $s=\sin$.

$$
{ }^{\mathrm{n}-1} \mathrm{~T}_{\mathrm{n}}=\left[\begin{array}{cccc}
c \theta_{n} & -s \theta_{n} & 0 & a_{n-1} \\
s \theta_{n} c \alpha_{n-1} & c \theta_{n} c \alpha_{n-1} & -s \alpha_{n-1} & -d_{n} s \alpha_{n-1} \\
s \theta_{n} s \alpha_{n-1} & c \theta_{n} s \alpha_{n-1} & c \alpha_{n-1} & d_{n} c \alpha_{n-1} \\
0 & 0 & 0 & 1
\end{array}\right]
$$

For the designed SCARA like Fig.1 and Table.1, the end machine is the axis of the fourth rod. The relation of the end machine with the first rod can be described as ${ }^{0} \mathrm{~T}_{4}={ }^{0} \mathrm{~T}_{1}{ }^{1} \mathrm{~T}_{2}{ }^{2} \mathrm{~T}_{3}{ }^{3} \mathrm{~T}_{4}$. The transformation of the designed SCARA is shown as equation (2) that in this equation $c=\cos \theta$ and $s=\sin \theta$. 


$$
{ }^{0} \mathrm{~T}_{1}=\left[\begin{array}{cccc}
c_{1} & -s_{1} & 0 & 0 \\
s_{1} & c_{1} & 0 & 0 \\
0 & 0 & 1 & d_{1} \\
0 & 0 & 0 & 1
\end{array}\right] \quad{ }^{1} \mathrm{~T}_{2}=\left[\begin{array}{cccc}
c_{2} & -s_{2} & 0 & l_{1} \\
s_{2} & c_{2} & 0 & 0 \\
0 & 0 & 1 & 0 \\
0 & 0 & 0 & 1
\end{array}\right] \quad{ }^{2} \mathrm{~T}_{3}=\left[\begin{array}{cccc}
1 & 0 & 0 & l_{2} \\
0 & -1 & 0 & 0 \\
0 & 0 & -1 & -d_{3} \\
0 & 0 & 0 & 1
\end{array}\right] \quad{ }^{3} \mathrm{~T}_{4}=\left[\begin{array}{cccc}
c_{4} & -s_{4} & 0 & 0 \\
s_{4} & c_{4} & 0 & 0 \\
0 & 0 & 1 & 0 \\
0 & 0 & 0 & 1
\end{array}\right]
$$

\subsection{Robot inverse kinematics analysis\&promotion}

The result of robot inverse kinematics is good or not that will affect the performance and precision of robot locus and control effect. On choosing inverse kinematics solution, it is important to adopt appropriate standards, constraint condition and criterion for judgment to get good results.

Criterion for judgment usually has such demands as follows.

(1) Robot corner range demand. Because robot mechanical limit, robot arm can not move or rotate in the full scope of the arm. Every rotating axis can only move in the limit condition:

$$
q_{i l} \leq q_{i} \leq q_{i h}, i=1,2, \ldots, n
$$

In the equation (1), $q_{i l}$ is the smallest angle of the $i$ axis, $q_{i h}$ is the biggest angle of the $i$ axis. This condition must be observed.

(2) Satisfy minimum position error demand. There will be position or locus error when robot in moving. If the error is bigger, the robot can not be used in some working environment. So there will be a certain error value that the robot moving position must obey:

$$
\left\|p_{w}-p_{w, d e s}\right\| \leq \varepsilon
$$

The $p_{w}$ is the real moving position, and $p_{w, d e s}$ is the designed moving position. This demand has high application value but it is hard to measure the position value in reality.

(3) Compliance requirements. Compliance requires that the angle of each axis changes smoothly and the angle will not changes suddenly. This can ensure that the motion shock of SCARA is small.

$$
\Delta \mathrm{A}=\left\|\{\mathrm{A}\}-\left\{\mathrm{A}_{\text {cur }}\right\}\right\| \leq k
$$

In equation (5), $\mathrm{A}$ is the angle in the next posture and $\mathrm{A}_{\mathrm{cur}}$ is the angle in the current posture. The $k$ is a fixed small angle we need.

(4) Moving the small arm as many as you can and moving the big arm as little as possible. The small arm is lighter and it can move faster and need littler energy than the big arm.

\subsection{Singularity analysis of SCARA}

Singular configuration is the inherent character of the Robot machine. When SCARA moves in the singular configuration, the Jacobian matrix will be a singular matrix. The singular matrix has not inverse matrix, so the inverse solution and control algorithm of SCARA can not be get in this time. When the SCARA moves in high speed and high precision, the singularity analysis must be needed.

The definition of Jacobian matrix is a matrix from angular velocity or joint velocity to space velocity. The motion equation of SCARA is described as equation (6), in which $r$ is the space axis and $\theta$ is the joint axis.

$$
r=f(q)
$$

If take the derivative of this equation, the equation (14) can be changed as equation (7).

$$
\dot{r}=J(\mathrm{q}) \dot{q}
$$

In this equation, $\dot{r}$ is the space velocity of end effector and $\dot{q}$ is the joint velocity of the SCARA. In equation (5). The $J(q)$ is a Jacobian matrix, in which the line number equals the motion dimension, and the column number equals the joint number of SCARA. In general, the velocity of rigid body is in three-dimensional space, in which line velocity $v$ and angular velocity $w$ of rigid body are three dimensional vector. So generalized velocity $r$ is a six dimensional column vector and Jacobian matrix of SCARA is a $6 * 4$ matrix, in which the line elements $1,2,3$ represents the line 
velocity transformation and column elements 4, 5, 6 represents the column velocity transformation of the end effector. So the Jacobian matrix can be written as partitioned matrix like equation (8). In equation (8), $v=\left[v_{x} v_{y} v_{z}\right]$ is velocity vector of axis $x, y, z$.

$$
\left[\begin{array}{c}
\mathrm{v} \\
w
\end{array}\right]=\left[\begin{array}{c}
J_{v 1} \ldots J_{v 4} \\
J_{w 1} \ldots J_{w 4}
\end{array}\right]\left[\begin{array}{c}
\dot{q}_{1} \\
\vdots \\
\dot{q}_{4}
\end{array}\right]
$$

By the knowledge of robot kinematics, the position of end effector $\mathrm{P}_{\mathrm{x}}, \mathrm{P}_{\mathrm{y}}, \mathrm{P}_{\mathrm{z}}$ can be represented by the joint variant like equation (9).

$$
\mathrm{v}_{x}=\frac{d p_{x}}{d t}=\frac{\partial p_{x}}{\partial q_{1}} \dot{q}_{1}+\frac{\partial p_{x}}{\partial q_{2}} \dot{q}_{2}+\ldots \frac{\partial p_{x}}{\partial q_{n}} \dot{q}_{n}=\left[\frac{\partial p_{x}}{\partial q_{1}} \ldots \frac{\partial p_{x}}{\partial q_{n}}\right]\left[\begin{array}{c}
\dot{q}_{1} \\
\vdots \\
\dot{q}_{n}
\end{array}\right.
$$

In equation(9), $J_{v i x}=\frac{\partial P_{x}}{\partial q_{i}}, J_{v i y}=\frac{\partial P_{y}}{\partial q_{i}}, J_{v i z}=\frac{\partial P_{z}}{\partial q_{i}}$. From this, we can conclude the results in equation (10).

$$
J_{v i}=\left[\begin{array}{lll}
\frac{\partial p_{x}}{\partial q_{i}} & \frac{\partial p_{y}}{\partial q_{i}} & \frac{\partial p_{z}}{\partial q_{i}}
\end{array}\right]^{T}
$$

When the transformation function is an angular velocity and the joint is a prismatic joint, the function can be written as the equation (11).

$$
J_{w}=\left[\begin{array}{lll}
0 & 0 & 0
\end{array}\right]^{T}
$$

When the joint $i$ is a rotation joint, the function can be written as the equation (12) vs. the angular velocity of end effector.

$$
J_{w}=Z_{i}
$$

$\mathrm{Z}_{\mathrm{i}}$ is the unit vector of joint $\mathrm{i}$. According to equation (8) to (10), the Jacobi matrix of SCARA can be written as equation (13).

$$
J_{i}=\left[\begin{array}{c}
J_{w} \\
0
\end{array}\right]
$$

The third joint of SCARA is a prismatic joint and the other joints are rotation joints. From the kinematics results of SCARA like equation (7), the equation (14) can be get.

$$
\left\{\begin{array}{c}
p_{x}=c_{1} c_{2} l_{2}-s_{1} s_{2} l_{2}+c_{1} l_{1} \\
p_{y}=c_{2} s_{1} l_{2}+c_{1} s_{2} l_{2}+s_{1} l_{1} \\
p_{z}=d_{1}-d_{3}
\end{array}\right\}
$$

From equation (14), differentiate the end position component to the joint variable and the equation (15) can be got.

$$
\left\{\begin{array}{l}
\frac{\partial p_{x}}{\partial \theta_{1}}=-s_{1} c_{2} l_{2}+c_{1} s_{2} l_{2}-s_{1} l_{1} \\
\frac{\partial p_{y}}{\partial \theta_{1}}=-c_{1} c_{2} l_{2}-s_{1} s_{2} l_{2}-c_{1} l_{1} \\
\frac{\partial p_{z}}{\partial \theta_{1}}=0
\end{array}\right\} ;\left\{\begin{array}{l}
\frac{\partial p_{x}}{\partial \theta_{2}}=-c_{1} s_{2} l_{2}+s_{1} c_{2} l_{2} \\
\frac{\partial p_{y}}{\partial \theta_{2}}=-s_{1} s_{2} l_{2}-c_{1} c_{2} l_{2} \\
\frac{\partial p_{z}}{\partial \theta_{2}}=0
\end{array}\right\} ;\left\{\begin{array}{l}
\frac{\partial p_{x}}{\partial d_{3}}=0 \\
\frac{\partial p_{y}}{\partial d_{3}}=0 \\
\frac{\partial p_{z}}{\partial d_{3}}=-1
\end{array}\right\} ;\left\{\begin{array}{l}
\frac{\partial p_{x}}{\partial \theta_{3}}=0 \\
\frac{\partial p_{y}}{\partial \theta_{3}}=0 \\
\frac{\partial p_{z}}{\partial \theta_{3}}=0
\end{array}\right\}
$$

According to transformation matrix of adjacent link rod member, the $\mathrm{Z}_{\mathrm{i}}$ axis results of link rod axis is in equation (16).

$$
Z_{1}=\left[\begin{array}{l}
0 \\
0 \\
1
\end{array}\right] ; Z_{2}=\left[\begin{array}{l}
0 \\
0 \\
1
\end{array}\right] ; Z_{3}=\left[\begin{array}{l}
0 \\
0 \\
0
\end{array}\right] ; Z_{4}=\left[\begin{array}{c}
0 \\
0 \\
-1
\end{array}\right]
$$


By the analysis above and using motion vector differentiation method, the Jacobia matrix of the SCARA can be written in equation (17).

$$
J_{4}^{0}=\left[\begin{array}{cccc}
-s_{1} c_{2} l_{2}+c_{1} s_{2} l_{2}-s_{1} l_{1} & -c_{1} s_{2} l_{2}+s_{1} c_{2} l_{2} & 0 & 0 \\
-c_{1} c_{2} l_{2}-s_{1} s_{2} l_{2}-c_{1} l_{1} & -s_{1} s_{2} l_{2}-c_{1} c_{2} l_{2} & 0 & 0 \\
0 & 0 & 1 & 0 \\
0 & 0 & 0 & 0 \\
0 & 0 & 0 & 0 \\
1 & 1 & 0 & -1
\end{array}\right]
$$

\section{Experiment and Conclusion}

When SCARA is in work, it will be influenced by a lot of elements like the deviation between real load and ideal load, the dead zone of transimission gear, and friction force changes by temperature and moving velocity changes etc. In designing the SCARA, all the elements can not be full considered. Even all the elements have been considered in the controller, the disturb elements can not be valued exactly. Because the disturbed value exists in real work, the moving locus of SCARA will not agree with the designed locus according to the theoretical model. If the deviation is too large, the precision of the SCARA will lost and even dangerous will arise. So a robust controller must be designed in order to satisfy the need if even the SCARA face some uncertain interference.

In this paper, an embedded controller of SCARA based on ARM is designed. In this controller, four type functions have been realized. First, geometric modeling of SCARA use D-H method is finished in the controller, with analyzing \& solving SCARA forward kinematics and inverse kinematics. Second, linear interpolation and circular interpolation algorithm of SCARA in cartesian coordinate system are finished. Three polynomial interpolation and cubic spline interpolation in joint space are also finished. Based on such fruits, PTP (point to point) movement and CP (continuous path) movement locus plan can be fulfilled in the controller. Third, the real-time control software of SCARA based on uC/OS-II is finished on the ARM (Acorn RISC Machine) hardware platform. The controller is working well with the mechanical machine of SCARA by the PTP, CP and other velocity-precision test experiments. The SCARA can be programmed by computer, touch pendant etc and can connect the smart camera to finish image grasping work.

The SCARA has good work speed, precision, degree of linearity etc. The beat test of SCARA is done, that the SCARA moves from A point to B point about $300 \mathrm{~mm}$ and the lift distance is $25 \mathrm{~mm}$ like Figure 2. The beat test is done with the load 1, 2 and $3 \mathrm{~kg}$.

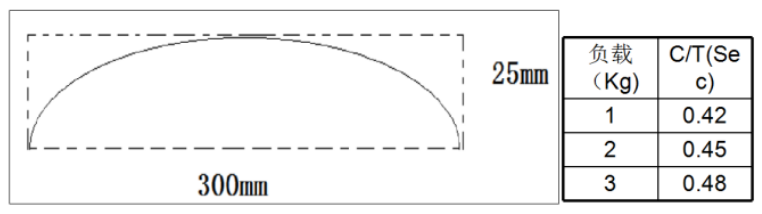

Figure 2 The beat test locus

By test with the camera, the SCARA is working in image distinguishing and grasping the micro electron device with the device size is about $10 * 8 \mathrm{~mm}$. The work scene is shown in Figure 3.

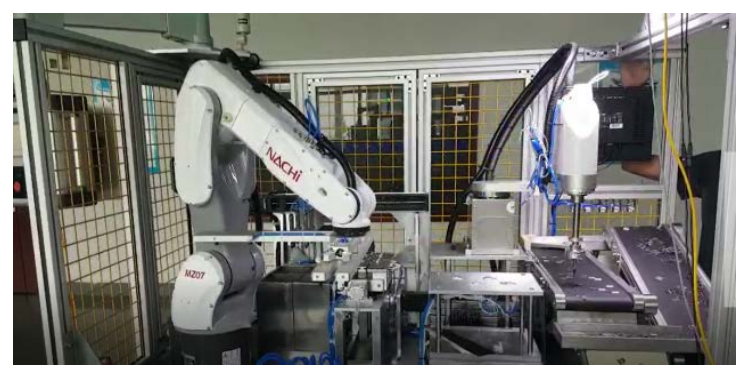

Figure 3 The working scene of SCARA micro grasping 


\section{Acknowledgments}

Thanks for Supported by National Natural Science Foundation of China (No. 61473200). And thanks for our research team.

\section{References}

[1] Khairudin M, Morhamed Z, Husain AR. Dynamic Model and Robust Control of Flexible Link Robot Manipulator[J]. TELKOMNIKA Indonesian Journal of Electrical Engineering, 2011, 9(2): 279-286.

[2] Serdar Kucuk, Zafer Bingul. The Inverse Kinematics Solutions of Industrial Robot Manipulators [J]. IEEE Journal of Robotics and Automation, IEEE (S0-7803-8599-3),2004:274279.

[3] S. Elgazzar. Efficient kinematic transformations for the PUMA 560 robot [J]. IEEE Journal of Robotics and Automation,2005,1 (3):142-151.

[4] Hartenberg R. S., Denavit J. A Kinematic Notation for Lower-Pair Mechanisms Based on Matrices [J]. Journal of Applied Mechanics, vol.21, no.5, pp. 215-221, 1995.

[5] Tolu, S., et al., Dynamics Model Abstraction Scheme Using Radial Basis Functions[J]. Journal of Control Science and Engineering, 2012.1-11. 